\title{
Classification of categories with matrices of coefficient 2 and order $n$
}

\author{
Samer Allouch ${ }^{\mathrm{a}, \mathrm{b}}$ and Carlos Simpson ${ }^{\mathrm{c}}$ \\ ${ }^{a}$ King Abdullah University of Science and Technology, Thuwal, \\ Saudi Arabia(KAUST) \\ ${ }^{b}$ Lebanese-French University of Technology and Applied Sciences , \\ Lebanon(ULF) \\ ${ }^{c}$ C.N.R.S, Laboratory J.A. Dieudonné, Université Côte d'Azur, \\ France
}

October 23, 2017

\begin{abstract}
In this paper, we present the categories associated to the square matrix with coefficients 2 of order 3 . The family of categories associated to these matrices has 5 isomorphism classes.

In the case of a matrix of order higher than 3 , we only demonstrate upper and lower bounds for the number of associated categories.
\end{abstract}

KEYWORDS: Finite categories; reduced category; category of the square matrix 2 . 


\section{Introduction}

Category theory was developed by mathematicians Samuel Eilenberg and Saunders Mac Lane then propagated by Grothendieck and his school in the sixties. This abstract theory allows us to generalize the concept of algebraic structures and has become an essential tool in algebraic geometry and algebraic topology.

Numerous studies have focused on the theory of categories, but less focus had been given to the finite categories. Recently however, finite categories have attracted significant attention. Leinster and Berger [4, 13] have studied the matrices corresponding to their finite categories, counting the numbers of morphisms between different objects. Fiore and Lück [8], have obtained necessary conditions for developability of a finite complex of groups from an action of a finite group on a finite category without loops.

The enumeration and classification of associative structures in general is a difficult problem, see for example [15]. In the case of finite categories, the corresponding matrix can provide an interesting invariant to help in the classification. In our previous paper [3], we presented necessary and sufficient conditions on the matrix to obtain at least one finite category.

A next question is: how many are there? The basic idea for going from the matrix towards a classification, is that if certain coefficients are small then it should provide additional information. One of the most initial cases is to study the finite categories with a matrix of coefficients 2 of order $n$, that is to say categories possessing exactly two morphisms between each pair of objects. The objective of this work is to consider the classification and counting of these categories. We proceed to start with the orders $n=2$ and $n=3$, then after this sequence is completed to look at a general order.

We say that a matrix 2 of order $n$ denoted by $M_{2}^{n}$ is a square matrix of order $n$ whose coefficients are all equal and have the value 2 . Next by Leinster 
and Berger [4] and our previous work [3] these kinds of matrices always admit associated categories.

For the classification it suffices to consider categories that don't have distinct pairs of isomorphic objects, such a category is said to be reduced.

We denote by $\operatorname{Card}\left(M_{2}^{n}, r\right)$ the cardinality of the set of all reduced categories that are associated with $M_{2}^{n}$ up to isomorphism and $\operatorname{Card}\left(M_{2}^{n}, r, o\right)$ the same for the ordered categories (provided with a total order or equivalently a numbering on the set of objects). In this paper we determine the exact value of $\operatorname{Card}\left(M_{2}^{n}, r\right)$ for $n=1,2,3$ and for $n>3$ we find an upper bound and lower bound for $\operatorname{Card}\left(M_{2}^{n}, r\right)$.

For $n=1$ there are two isomorphism types, and for $n=2$ just one isomorphism type of such categories.

For $n=3$ there are five isomorphism types. For the internal compositions $X^{k j} \circ Y^{j i}$ we first have the following properties:

$$
X^{i j} \circ Y^{j i}=B^{i i}, \quad B^{k j} \circ Y^{j i}=X^{k j} \circ B^{j i}=B^{k i} .
$$

For the other forms of composition and specifically the forms $A^{k j} \circ A^{j i}$ with $i \neq j \neq k$, the five categories of $M_{2}^{3}$ are characterized by the compositions in the following table:

\begin{tabular}{|c|c|c|c|c|c|c|}
\hline triple & comp & $\mathscr{A}_{1}$ & $\mathscr{A}_{2}$ & $\mathscr{A}_{3}$ & $\mathscr{A}_{4}$ & $\mathscr{A}_{5}$ \\
\hline$(213)$ & $A^{31} \circ A^{12}=$ & $B^{32}$ & $B^{32}$ & $B^{32}$ & $B^{32}$ & $A^{32}$ \\
\hline$(312)$ & $A^{21} \circ A^{13}=$ & $B^{23}$ & $B^{23}$ & $B^{23}$ & $B^{23}$ & $B^{23}$ \\
\hline$(123)$ & $A^{32} \circ A^{21}=$ & $B^{31}$ & $B^{31}$ & $B^{31}$ & $B^{31}$ & $B^{31}$ \\
\hline$(321)$ & $A^{12} \circ A^{23}=$ & $B^{13}$ & $B^{13}$ & $B^{13}$ & $A^{13}$ & $A^{13}$ \\
\hline$(231)$ & $A^{13} \circ A^{32}=$ & $B^{12}$ & $B^{12}$ & $A^{12}$ & $B^{12}$ & $B^{12}$ \\
\hline$(132)$ & $A^{23} \circ A^{31}=$ & $B^{21}$ & $A^{21}$ & $A^{21}$ & $A^{21}$ & $A^{21}$ \\
\hline
\end{tabular}

Table 1: Composition for three objects

Once these classifications are done, we turn to the problem of getting general bounds on $\operatorname{Card}\left(M_{2}^{n}, r\right)$. In order to understand the growth rate as a function 
of $n$, put

$$
\sigma:=\lim _{n \rightarrow \infty} \sup \frac{\log \left(\operatorname{Card}\left(M_{2}^{n}, r\right)\right)}{n^{3}} .
$$

We obtain the bounds

$$
\frac{\log (2)}{27} \leq \sigma \leq \frac{\log (18)}{6}
$$

This uses the classification for $n=3$, notably the fact that $\operatorname{Card}\left(M_{2}^{3}, r, o\right)=18$.

We would like to thank Abdelkrim Aliouche, Persi Diaconis, and Tom Leinster for conversations inspiring the start of this work.

\section{Preliminaries}

We need to construct and recall some definitions and notations concerning the categories of matrix $M_{2}^{n}$.

\subsection{Categories of matrix 2 of order $n$}

Definition 2.1 A finite category is a category with a finite set of objects and a finite set of morphisms. In this case we have several methods for numbering the objects and the morphisms, moreover the order of this category is defined to be the cardinal of the set of objects.

Let $\mathscr{A}$ be a finite category of order $n$, assume that $O b(\mathscr{A})=\left\{x_{1}, x_{2}, \ldots, x_{n}\right\}$ and we construct an order relation over $O b(\mathscr{A})$ that is given by:

$$
x_{i}<x_{j} \Longleftrightarrow i<j
$$

Therefore $O b(\mathscr{A})$ becomes a ordered set, and $\mathscr{A}$ is called ordered category [2]. The associated matrix is by definition $M(i, j)=\left|\mathscr{A}\left(x_{i}, x_{j}\right)\right|$. 
Throughout this work, we consider $\mathscr{A}$ a category associated to $M_{2}^{n}$, where $M_{2}^{n}$ is the square matrix of order $n$ which is defined by:

$$
M_{2}^{n}=\left(\begin{array}{cccc}
2 & 2 & \ldots & 2 \\
2 & 2 & \ldots & 2 \\
\vdots & \vdots & \ddots & \vdots \\
2 & 2 & \ldots & 2
\end{array}\right)
$$

Therefore, each morphism set $\mathscr{A}\left(x_{i}, x_{j}\right)$ has two elements.

According to (1) we note that the symbol of an object $x_{i}$ and its index $i$ play the same role, so from now on we take $O b(\mathscr{A})=\{1,2, \ldots, n\}$, and for every $i \neq j$ the morphisms of $\mathscr{A}$ are given by [1]: $\mathscr{A}(i, i)=\left\{A^{i i}, B^{i i}\right\}$ and $\mathscr{A}(i, j)=\left\{A^{j i}, B^{j i}\right\}$, where $A^{i i}$ is the identity morphism $i d_{i i}$ and $A \neq B$.

In order to avoid ambiguity the notation $\left(X^{j i}\right)$ is designed to make visible the information of sources and targets of morphisms during the calculations.

Definition 2.2 We say that $\mathscr{A}$ is a reduced category if there does not exist any isomorphism between two distinct objects.

If $\mathscr{A}$ is a reduced category and $i, j$ two distinct objects, then

$$
B^{j j} \circ A^{j i}=B^{j j} \circ B^{j i}=A^{j i} \circ B^{i i}=B^{j i} \circ B^{i i} .
$$

This will be proved in the next section.

In view of these properties, we may therefore fix the notation that

$$
B^{j i}:=B^{j j} \circ A^{j i}=B^{j j} \circ B^{j i}=A^{j i} \circ B^{i i}=B^{j i} \circ B^{i i} .
$$

The morphism $A^{j i}$ is the other one not equal to this.

\subsection{Set of categories and morphisms of the matrix $M_{2}^{n}$}

To classify the categories with matrix $M_{2}^{n}$, we need to define some terms.

- $\operatorname{Card}\left(M_{2}^{n}\right)$ is the cardinal of the set of isomorphism classes of categories 
associated to $M_{2}^{n}$.

- $\operatorname{Card}\left(M_{2}^{n}, r\right)$ is the cardinal of the set of isomorphism classes of reduced categories associated to $M_{2}^{n}$.

- $\operatorname{Card}\left(M_{2}^{n}, r, o\right)$ is the cardinal of the set of classes of reduced and ordered categories associated to $M_{2}^{n}$, up to isomorphism preserving the ordering.

These terms readily lead to the following property:

$$
0 \neq \frac{\operatorname{Card}\left(M_{2}^{n}, r, o\right)}{n !} \leq \operatorname{Card}\left(M_{2}^{n}, r\right) \leq \operatorname{Card}\left(M_{2}^{n}, r, o\right) .
$$

Let $\mathscr{A}$ be a reduced category of $M_{2}^{n}$ such that its objects are denoted $\{1, \ldots, n\}$. We note by $[i, j, k]$ the set of all morphisms among three objects $i, j$ and $k$. If $(i \neq j \neq k \neq i)$, we say $[i, j, k]$ is distinct. We denote the composition $A^{i j} \circ A^{j k}$ by $\langle i, j, k\rangle$.

By the theorem (3.8) below, we will know the formulas for composition within any non-distinct triple, namely $A^{i i}$ are identities, any composition with a morphism $B$ will be a morphism $B$, and compositions with the same source and target are $B$, so it remains to study the formulas for composition within a distinct triple $(i, j, k)$.

Definition 2.3 Let us define the function $\alpha:\{(i, j, k) \mid i, j, k \in \mathrm{Ob}(\mathscr{A})\} \rightarrow\{0,1\}$ by

$$
\alpha:(i, j, k) \quad \longmapsto \quad \alpha((i, j, k))=\left\{\begin{array}{ccc}
0 & \text { if } & \langle i, j, k\rangle=B^{i k} \\
1 & \text { if } & \langle i, j, k\rangle=A^{i k}
\end{array}\right.
$$

We say that $\mathscr{A}$ is exceptional at a distinct triple $(i j k)$ if $\alpha(i, j, k)=1$. 


\section{Composition in the categories of $M_{2}^{n}$}

Pick $\mathscr{A}$ a reduced category associated to $M_{2}^{n}$ with $O b(\mathscr{A})=\{1, \ldots, n\}$, where $n \geq 2$. For every $i \neq j$ in $O b(\mathscr{A})$, as above the morphisms are denoted by $\mathscr{A}(i, j)=\left\{A^{j i}, B^{j i}\right\}$. We follow the convention that $A^{i i}=i d_{i}$.

\subsection{Composition of two morphisms for two distinct ob- jects}

Let $X, Y \in\{A, B\}$, we denote by $X^{\prime}$ and $Y^{\prime}$ the morphisms otherwise than $X$ and $Y$ for example:

$$
X^{\prime}= \begin{cases}A & \text { if } X=B \\ B & \text { if } X=A\end{cases}
$$

Theorem 3.1 There exist at least two morphisms $X, Y \in\{A, B\}$ such that $Y^{i j} \circ X^{j i}=B^{i i}$.

\section{Proof:}

To the contrary, assume that $Y^{i j} \circ X^{j i}=A^{i i}=i d_{i}$ for all $X, Y \in\{A, B\}$, then

$$
\left[A^{i j} \circ A^{j i}\right] \circ B^{i i}=i d_{i} \circ B^{i i}=B^{i i}=A^{i j} \circ\left[A^{j i} \circ B^{i i}\right]=A^{i j} \circ X^{j i}=A^{i i} .
$$

This is a contradiction, since $B^{i i} \neq A^{i i}$.

Theorem 3.2 If we have $B^{i i} \circ B^{i i}=A^{i i}=i d_{i}$ then for any $X, Y \in\{A, B\}$ and any $j \neq i$, we get the following properties:

1. $X^{j i} \circ B^{i i}=\left(X^{\prime}\right)^{j i}$ and $B^{i i} \circ Y^{i j}=\left(Y^{\prime}\right)^{i j}$

2. $Y^{i j} \circ X^{j i}=\left(Y^{\prime}\right)^{i j} \circ\left(X^{\prime}\right)^{j i} \neq\left(Y^{\prime}\right)^{i j} \circ X^{j i}=Y^{i j} \circ\left(X^{\prime}\right)^{j i}$

3. $B^{j j} \circ B^{j j}=A^{j j}=i d_{j}$.

\section{Proof:}

(1) 
If $X^{j i} \circ B^{i i}=\left(X^{\prime}\right)^{j i} \circ B^{i i}$ then,

$X^{j i} \circ\left[B^{i i} \circ B^{i i}\right]=X^{j i} \circ A^{i i}=X^{j i}=\left[X^{j i} \circ B^{i i}\right] \circ B^{i i}=\left[\left(X^{\prime}\right)^{j i} \circ B^{i i}\right] \circ B^{i i}=\left(X^{\prime}\right)^{j i}$.

This is a contradiction, since $X^{\prime} \neq X$, so $X^{j i} \circ B^{i i} \neq\left(X^{\prime}\right)^{j i} \circ B^{i i}$.

It remains to be shown that $X^{j i} \circ B^{i i}=\left(X^{\prime}\right)^{j i}$.

Suppose to the contrary that $X^{j i} \circ B^{i i}=X^{j i}$, then:

$\left[Y^{i j} \circ X^{j i}\right] \circ B^{i i}=Y^{i j} \circ\left[X^{j i} \circ B^{i i}\right]=Y^{i j} \circ X^{j i}$. But $Y^{i j} \circ X^{j i} \in\left\{A^{i i}, B^{i i}\right\}$,

so, we have two cases:

- If $Y^{i j} \circ X^{j i}=A^{i i}=i d_{i}$, then:

$\left[Y^{i j} \circ X^{j i}\right] \circ B^{i i}=B^{i i}=Y^{i j} \circ X^{j i}=A^{i i}$ which is impossible $\left(B^{i i} \neq A^{i i}\right)$.

- If $Y^{i j} \circ X^{j i}=B^{i i}$, then:

$\left[Y^{i j} \circ X^{j i}\right] \circ B^{i i}=B^{i i} \circ B^{i i}=A^{i i}=Y^{i j} \circ X^{j i}=B^{i i}$ which is impossible $\left(B^{i i} \neq A^{i i}\right)$.

In both cases we get a contradiction, therefore $X^{j i} \circ B^{i i}=\left(X^{\prime}\right)^{j i}$.

Finally, we find that:

$X^{j i} \circ B^{i i}=\left(X^{\prime}\right)^{j i}$ and $\left(X^{\prime}\right)^{j i} \circ B^{i i}=X^{j i}$.

Following the same ideas:

$B^{i i} \circ Y^{i j}=\left(Y^{\prime}\right)^{i j}$ and $B^{i i} \circ\left(Y^{\prime}\right)^{i j}=Y^{i j}$.

We have,

$\left[Y^{i j} \circ X^{j i}\right] \circ\left[B^{i i} \circ B^{i i}\right]=Y^{i j} \circ\left[X^{j i} \circ B^{i i}\right] \circ B^{i i}=\left[Y^{i j} \circ\left(X^{\prime}\right)^{j i}\right] \circ B^{i i}$

$\left[B^{i i} \circ B^{i i}\right] \circ\left[Y^{i j} \circ X^{j i}\right]=B^{i i} \circ\left[B^{i i} \circ Y^{i j}\right] \circ X^{j i}=B^{i i} \circ\left[\left(Y^{\prime}\right)^{i j} \circ X^{j i}\right]$ so,

$Y^{i j} \circ X^{j i}=\left[Y^{i j} \circ\left(X^{\prime}\right)^{j i}\right] \circ B^{i i}=B^{i i} \circ\left[\left(Y^{\prime}\right)^{i j} \circ X^{j i}\right]$.

Similarly,

$\left(Y^{\prime}\right)^{i j} \circ\left(X^{\prime}\right)^{j i}=\left[\left(Y^{\prime}\right)^{i j} \circ X^{j i}\right] \circ B^{i i}=B^{i i} \circ\left[Y^{i j} \circ\left(X^{\prime}\right)^{j i}\right]$.

There are two cases for $\left(Y^{\prime}\right)^{i j} \circ X^{j i}$ :

- If $\left(Y^{\prime}\right)^{i j} \circ X^{j i}=A^{i i}=i d_{i}$ then,

$$
Y^{i j} \circ X^{j i}=\left(Y^{\prime}\right)^{i j} \circ\left(X^{\prime}\right)^{j i}=B^{i i} \text { and }\left(Y^{\prime}\right)^{i j} \circ X^{j i}=A^{i i}=i d_{i}
$$


- If $\left(Y^{\prime}\right)^{i j} \circ X^{j i}=B^{i i}$ then,

$$
Y^{i j} \circ X^{j i}=\left(Y^{\prime}\right)^{i j} \circ\left(X^{\prime}\right)^{j i}=A^{i i}=i d_{i} \text { and }\left(Y^{\prime}\right)^{i j} \circ X^{j i}=B^{i i}
$$

Thus, $Y^{i j} \circ X^{j i}=\left(Y^{\prime}\right)^{i j} \circ\left(X^{\prime}\right)^{j i} \neq\left(Y^{\prime}\right)^{i j} \circ X^{j i}=Y^{i j} \circ\left(X^{\prime}\right)^{j i}$.

According to the theorem (3.1), there exist $X, Y \in\{A, B\}$ with $Y^{i j} \circ X^{j i}=B^{i i}$.

But, by the preceding argument,

$$
\text { if } B^{i i} \circ B^{i i}=A^{i i}=i d_{i} \Rightarrow\left\{\begin{array}{l}
X^{j i} \circ B^{i i}=\left(X^{\prime}\right)^{j i} \\
Y^{i j} \circ X^{j i}=\left(Y^{\prime}\right)^{i j} \circ\left(X^{\prime}\right)^{j i}=B^{i i} \\
\left(Y^{\prime}\right)^{i j} \circ X^{j i}=Y^{i j} \circ\left(X^{\prime}\right)^{j i}=A^{i i}=i d_{i}
\end{array}\right.
$$

Note that

$B^{j j} \circ X^{j i}=\left(X^{\prime}\right)^{j i}$, because if we take $B^{j j} \circ X^{j i}=X^{j i}$ then,

$$
X^{j i}\left[Y^{i j} \circ X^{j i}\right]=X^{j i} \circ B^{i i}=\left(X^{\prime}\right)^{j i}=\left[X^{j i} \circ Y^{i j}\right] \circ X^{j i} .
$$

Thus, $\left(X^{\prime}\right)^{j i}=\left[X^{j i} \circ Y^{i j}\right] \circ X^{j i}$.

If $X^{j i} \circ Y^{i j}=A^{j j}$ then, $\left(X^{\prime}\right)^{j i}=X^{j i}$ contradiction.

If $X^{j i} \circ Y^{i j}=B^{j j}$ then, $\left(X^{\prime}\right)^{j i}=B^{j j} \circ X^{j i}=X^{j i}$ contradiction.

In both cases we arrive at an impossibility, therefore $B^{j j} \circ X^{j i}=\left(X^{\prime}\right)^{j i}$.

In the same way, one shows that $B^{j j} \circ\left(X^{\prime}\right)^{j i}=X^{j i}$.

We come back to the proof that $B^{j j} \circ B^{j j}=A^{j j}=i d_{j}$.

Suppose instead that $B^{j j} \circ B^{j j}=B^{j j}$ then,

$$
\left(X^{\prime}\right)^{j i}=\left[B^{j j} \circ B^{j j}\right] \circ X^{j i}=B^{j j} \circ\left[B^{j j} \circ X^{j i}\right]=B^{j j} \circ\left(X^{\prime}\right)^{j i}=X^{j i} .
$$

This is a contradiction, so $B^{j j} \circ B^{j j}=A^{j j}=i d_{j}$.

Corollary 3.3 With the assumption $n \geq 2$, we have $B^{i i} \circ B^{i i}=B^{i i}$ for all $i$.

\section{Proof:}

To the contrary, suppose that $B^{i i} \circ B^{i i}=A^{i i}=i d_{i}$. According to the previous theorem, $B^{j j} \circ B^{j j}=A^{j j}$ for some $j \neq i$. But then if $X^{i j}$ and $Y^{j i}$ are any morphisms, their compositions $X^{i j} \circ Y^{j i}$ and $Y^{j i} \circ X^{i j}$ are either the identity or an invertible morphism ( $B^{i i}$ or $B^{j j}$ respectively), so $X^{i j}$ and $Y^{j i}$ are isomorphisms, 
contradicting the reduced property of $\mathscr{A}$.

Lemma 3.4 There exists a morphism $f \in \mathscr{A}(i, j)$ such that $B^{j j} \circ f=f$, $f \circ B^{i i}=f$, and for any $g \in \mathscr{A}(j, i)$ we have $f \circ g=B^{j j}$ and $g \circ f=B^{i i}$.

\section{Proof:}

Choose a morphism $X^{j i} \in \mathscr{A}(i, j)$ and set $f:=B^{j j} \circ X^{j i} \circ B^{i i}$. The previous corollary gives the first properties. For the second ones note that $f \circ g=$ $B^{j j} \circ(f \circ g)$ so this has to be $B^{j j}$.

By this lemma, there are only two cases for the types of morphisms:

$X^{j i} \circ B^{i i}, B^{j j} \circ X^{j i}, B^{i i} \circ Y^{i j}$ and $Y^{i j} \circ B^{j j}$ :

1. identity case, for example:

$$
X^{j i} \circ B^{i i}=X^{j i} \text { and }\left(X^{\prime}\right)^{j i} \circ B^{i i}=\left(X^{\prime}\right)^{j i}
$$

2. constant case, for example:

$$
X^{j i} \circ B^{i i}=\left(X^{\prime}\right)^{j i} \circ B^{i i} .
$$

Indeed, the case where composition with $B^{i i}\left(\operatorname{resp} . B^{j j}\right.$ ) interchanges $X^{i j}$ and $\left(X^{\prime}\right)^{i j}$ is ruled out.

Theorem 3.5 The morphisms $X^{j i} \circ B^{i i}, B^{j j} \circ X^{j i}, B^{i i} \circ Y^{i j}$ and $Y^{i j} \circ B^{j j}$ are in the constant case.

\section{Proof:}

Suppose for example that $X^{j i} \circ B^{i i}$ is in the identity case, so $X^{j i} \circ B^{i i}=X^{j i}$ and $\left(X^{\prime}\right)^{j i} \circ B^{i i}=\left(X^{\prime}\right)^{j i}$. We may assume that $X^{j i}$ is the morphism $f$ of Lemma

3.4. Thus, $Y^{i j} \circ X^{j i}=B^{i i}$ for any $Y^{i j}$. Now

$$
\begin{gathered}
\left(X^{\prime}\right)^{j i}=\left(X^{\prime}\right)^{j i} \circ B^{i i}=\left(X^{\prime}\right)^{j i} \circ\left(Y^{i j} \circ X^{j i}\right)=\left(\left(X^{\prime}\right)^{j i} \circ Y^{i j}\right) \circ X^{j i} \\
=\left[\text { either } A^{j j} \text { or } B^{j j}\right] \circ X^{j i}=X^{j i}
\end{gathered}
$$


a contradiction. This proves that $X^{j i} \circ B^{i i}$ is in the constant case. The other statements are the same.

Lemma 3.6 If $i \neq j$ and for any $X^{j i}$ and $Y^{i j}$ we have $Y^{i j} \circ X^{j i}=B^{i i}$ and $X^{j i} \circ Y^{i j}=B^{j j}$.

\section{Proof:}

Suppose $Y^{i j} \circ X^{j i}=A^{i i}$. Then if $\left(X^{\prime}\right)^{j i} \circ Y^{i j}=A^{j j}$ it gives that $Y^{i j}$ admits left and right inverses therefore $i$ and $j$ are isomorphic, impossible because $\mathscr{A}$ is a reduced category. Therefore we may assume $\left(X^{\prime}\right)^{j i} \circ Y^{i j}=B^{j j}$. We also have $X^{j i} \circ Y^{i j}=B^{j j}$. for the same reason. Then:

$\left(X^{\prime}\right)^{j i}=\left(X^{\prime}\right)^{j i} \circ\left[Y^{i j} \circ X^{j i}\right]=\left[\left(X^{\prime}\right)^{j i} \circ Y^{i j}\right] \circ X^{j i}=B^{j j} \circ X^{j i}=\left[X^{j i} \circ Y^{i j}\right] \circ X^{j i}=$ $X^{j i} \circ\left[Y^{i j} \circ X^{j i}\right]=X^{j i}$,

a contradiction. This shows the first statement, the other is similar.

Remark 3.7 Because of Theorem 3.5, the morphism $f$ of Lemma 3.4 is unique, and we will from now on fix the following equalities in our notational convention: $X^{j i} \circ B^{i i}=\left(X^{\prime}\right)^{j i} \circ B^{i i}=B^{j j} \circ X^{j i}=B^{j j} \circ\left(X^{\prime}\right)^{j i}=B^{j i}$, it is the morphism $f$.

\subsection{Composition of two morphisms for three distinct ob- jets}

Let $i, j$ and $k$ are three distinct objects in $O b(\mathscr{A})$. A main property is as follows.

Theorem 3.8 Using the convention of the remark (3.7) for morphisms, we have:

1. $B^{k j} \circ B^{j i}=A^{k j} \circ B^{j i}=B^{k j} \circ A^{j i}=B^{k i}$

2.

$$
\text { If } A^{k j} \circ A^{j i}=A^{k i} \Longrightarrow\left\{\begin{array}{c}
A^{j k} \circ A^{k i}=B^{j i} \\
\text { and } \\
A^{k i} \circ A^{i j}=B^{k j}
\end{array}\right.
$$




\section{Proof:}

(1)

We will show that:

$$
B^{k j} \circ B^{j i}=A^{k j} \circ B^{j i}=B^{k j} \circ A^{j i} .
$$

By the remark (3.7), we have:

$A^{k j} \circ B^{j j}=B^{k j} \circ B^{j j}=B^{k j}$ and $B^{j j} \circ A^{j i}=B^{j j} \circ B^{j i}=B^{j i}$

On the other hand,

$$
\begin{gathered}
{\left[B^{k j} \circ B^{j j}\right] \circ A^{j i}=B^{k j} \circ A^{j i}=B^{k j} \circ\left[B^{j j} \circ A^{j i}\right]=B^{k j} \circ B^{j i} \text {. Thus, }} \\
B^{k j} \circ A^{j i}=B^{k j} \circ B^{j i} .
\end{gathered}
$$

The other direction,

$$
\begin{gathered}
{\left[A^{k j} \circ B^{j j}\right] \circ B^{j i}=B^{k j} \circ B^{j i}=A^{k j} \circ\left[B^{j j} \circ B^{j i}\right]=A^{k j} \circ B^{j i} \text {. Thus, }} \\
B^{k j} \circ B^{j i}=A^{k j} \circ B^{j i} .
\end{gathered}
$$

Then, $(\mathrm{a})+(\mathrm{b})$ gives:

$B^{k j} \circ B^{j i}=A^{k j} \circ B^{j i}=B^{k j} \circ A^{j i}$.

Observe that

$B^{k j} \circ B^{j i}=B^{k j} \circ\left[B^{j i} \circ B^{i i}\right]=\left[B^{k j} \circ B^{j i}\right] \circ B^{i i}=\left[A^{k i}\right.$ or $\left.B^{k i}\right] \circ B^{i i}=B^{k i}$.

One must show the following implication:

$$
A^{k j} \circ A^{j i}=A^{k i} \Rightarrow\left\{\begin{array}{l}
A^{j k} \circ A^{k i}=B^{j i} \\
A^{k i} \circ A^{i j}=B^{k j}
\end{array}\right.
$$

According to (3.6) and (3.7), so we have:

$$
\begin{gathered}
{\left[A^{j k} \circ A^{k j}\right] \circ A^{j i}=B^{j j} \circ A^{j i}=B^{j i}=A^{j k} \circ\left[A^{k j} \circ A^{j i}\right]=A^{j k} \circ A^{k i} \text {. Thus, }} \\
A^{j k} \circ A^{k i}=B^{j i} .
\end{gathered}
$$

And

$$
\begin{gathered}
A^{k j} \circ\left[A^{j i} \circ A^{i j}\right]=A^{k j} \circ B^{j j}=B^{k j}=\left[A^{k j} \circ A^{j i}\right] \circ A^{i j}=A^{k i} \circ A^{i j} \text {. So, } \\
A^{k i} \circ A^{i j}=B^{k j} .
\end{gathered}
$$


In terms of Definition (2.3), part (2) of the theorem says that if $\mathscr{A}$ is exceptional at $(a b c)$ then it is not exceptional at $(a c b)$ or at $(b a c)$.

\section{Classification of categories of matrix $M_{2}^{n}$}

\subsection{Categories of matrix $M_{2}^{2}$}

\section{Lemma 4.1}

$$
\operatorname{Card}\left(M_{2}^{2}, r\right)=1
$$

Indeed: With the notations as fixed by the convention of remark (3.7), we can represent the only category $\mathscr{A}$ by the following composition for all $i, j \in\{1,2\}$ and $X, Y \in\{A, B\}, B^{i i} \circ B^{i i}=B^{i i}, X^{i j} \circ B^{j j}=B^{i j}, B^{i i} \circ X^{i j}=B^{i j}$ and $X^{i j} \circ Y^{j i}=B^{i i}$, therefore $\operatorname{Card}\left(M_{2}^{2}, r\right)=1$.

\subsection{Categories of matrix $M_{2}^{3}$}

For $n=3$ we look at categories associated to the matrix $M_{2}^{3}$.

\section{Theorem 4.2}

$$
\operatorname{Card}\left(M_{2}^{3}, r\right)=5 \text {. }
$$

\section{Proof:}

Let $i, j$ and $k$ are three objects in $\{1,2,3\}$, we going on to study the morphisms of the form $Y^{k j} \circ X^{j i}$.

In the cases of $(j=k)$ or $(i=j)$, we take $Y^{j j}=B^{j j}$ or $\left(X^{i i}=B^{i i}\right)$, because the other case is the identity.

1. If $i=j=k$ so $Y^{k j} \circ X^{j i}=B^{i i} \circ B^{i i}=B^{i i}$ see (3.3)

2. If $(i \neq j=k)$ or $(i=j \neq k)$ then the remark (3.7) gives the following:

$$
Y^{k j} \circ X^{j i}=B^{j j} \circ X^{j i}=B^{j j} \circ\left(X^{\prime}\right)^{j i}=B^{j i}
$$




$$
Y^{k j} \circ X^{j i}=Y^{k j} \circ B^{j j}=\left(Y^{\prime}\right)^{k j} \circ B^{j j}=B^{k j}
$$

3. If $i=k$ then $X^{k j} \circ Y^{j i}=B^{k i}$.

4. If $i \neq j \neq k \neq i$, so $B^{k j} \circ B^{j i}=A^{k j} \circ B^{j i}=B^{k j} \circ A^{j i}=B^{k i}$ see (3.8), thus it remains to study these morphisms $A^{k j} \circ A^{j i}$ that have four cases:

(a) If $A^{31} \circ A^{12}=B^{32}$ and $A^{21} \circ A^{13}=B^{23}$, and to avoid the repetition, we can reduce the possibilities of the morphisms $A^{23} \circ A^{31}$ and $A^{13} \circ$ $A^{32}$ on the following cases:

i. Where $A^{23} \circ A^{31}=B^{21}$ and $A^{13} \circ A^{32}=B^{12}$ so, we have four cases which are represented by: $A^{32} \circ A^{21} \in\left\{A^{31}, B^{21}\right\}$ and $A^{12} \circ A^{23} \in\left\{A^{13}, B^{13}\right\}$, isomorphic to $\mathscr{A}_{1}, \mathscr{A}_{2}$ or $\mathscr{A}_{3}$.

ii. Where $A^{23} \circ A^{31}=A^{21}$ and $A^{13} \circ A^{32}=B^{12}$ so, we have two cases which are represented by: $A^{32} \circ A^{21}=B^{31}$ and $A^{12} \circ A^{23} \in\left\{A^{13}, B^{13}\right\}$. See (3.8). They are isomorphic to $\mathscr{A}_{1}$ and $\mathscr{A}_{4}$.

iii. Where $A^{23} \circ A^{31}=B^{21}$ and $A^{13} \circ A^{32}=A^{12}$ so, we have two cases which are represented by: $A^{32} \circ A^{21} \in\left\{A^{31}, B^{31}\right\}$ and $A^{12} \circ A^{23}=B^{13}$. See (3.8), they are again isomorphic to $\mathscr{A}_{1}$ and $\mathscr{A}_{4}$.

With this case we get 4 distinct categories $\mathscr{A}_{1}, \mathscr{A}_{2}, \mathscr{A}_{3}$ and $\mathscr{A}_{4}$ that are not isomorphic among themselves, see (Table 1).

(b) If $A^{31} \circ A^{12}=A^{32}$ and $A^{21} \circ A^{13}=A^{23}$, according to the theorem (3.8) we have:

$$
A^{13} \circ A^{32}=B^{12} \quad A^{32} \circ A^{21}=B^{31}
$$




$$
A^{12} \circ A^{23}=B^{13} \quad A^{23} \circ A^{31}=B^{21} .
$$

So we get one category that is isomorphic with $\mathscr{A}_{3}$ see (Table 1 ).

(c) If $A^{31} \circ A^{12}=A^{32}, A^{21} \circ A^{13}=B^{23}$ which give:

$A^{13} \circ A^{32}=B^{12}$ and $A^{32} \circ A^{21}=B^{31}$, see (3.8), so we have 4 cases:

i. If $A^{12} \circ A^{23}=B^{13}, A^{23} \circ A^{31}=B^{21}$, so we are getting a new category, but it looks like the category $\mathscr{A}_{2}$ see (Table 1 ).

ii. If $A^{12} \circ A^{23}=B^{13}, A^{23} \circ A^{31}=A^{21}$, so we get a new category, but it looks like the category $\mathscr{A}_{4}$ see (Table 1$)$.

iii. If $A^{12} \circ A^{23}=A^{13}, A^{23} \circ A^{31}=B^{21}$, so we are getting a new category, but it looks like the category $\mathscr{A}_{4}$ see (Table 1 ).

iv. If $A^{12} \circ A^{23}=A^{13}, A^{23} \circ A^{31}=A^{21}$, so we are getting a new category $\mathscr{A}_{5}$ which is defined in the introduction see (Table 1 ).

(d) If $A^{31} \circ A^{12}=B^{32}, A^{21} \circ A^{13}=A^{23}$ this case looks like the previous case.

Finally, in all these cases we are getting five categories $\mathscr{A}_{1}, \mathscr{A}_{2}, \mathscr{A}_{3}, \mathscr{A}_{4}$ and $\mathscr{A}_{5}$. These categories aren't isomorphic among themselves because of the function $\alpha$ that is defined above (2.3) which is an invariant, and the isomorphism classes (up to permutations) of their functions $\alpha$ are different as may be seen from the characterizations given in the proof of Theorem 5.2 below.

\section{$5 \quad$ The bounds for $\mathcal{C}$ ard $\left(M_{2}^{n}, r\right)$}

Now let us look for the bounds of the value $\mathcal{C}$ ard $\left(M_{2}^{n}, r\right)$, therefore we use the function $\alpha$ which is defined above see (2.3) and other terms that are defined below.

As a result of the above, we shall focus on the case of $n \geq 4$ i.e. we are interested to study the bounds of $\mathcal{C}$ ard $\left(M_{2}^{n}, r\right)$ where $n>3$. 


\subsection{Function $\alpha$ and Composition of Morphisms}

Theorem 5.1 If $\alpha:\{1, \ldots, n\}^{3} \rightarrow\{0,1\}$ is a function satisfying the conditions described below, then it corresponds (as in Definition 2.3) to a single category and all reduced categories come from these categories for functions $\alpha$.

Thus, the classification of reduced categories is equivalent to the classification of functions $\alpha$ that satisfy the following conditions.

1. For $j \neq i, \alpha(i, j, i)=0$, whereas $\alpha(i, i, i)=\alpha(i, i, j)=\alpha(i, j, j)=1$.

2. Let $[i, j, k]$ be a distinct triple then we have the following implication:

$$
\text { If } \alpha((i, j, k))=1 \Longrightarrow\left\{\begin{array}{c}
\alpha((i, k, j))=0 \\
\text { and } \\
\alpha((j, i, k))=0
\end{array}\right.
$$

3. Let $i, j, h$ and $k$ be distinct indices then we have the following implication:

$$
\left\{\begin{array} { c } 
{ \alpha ( ( i , j , k ) ) = 1 } \\
{ \text { and } } \\
{ \alpha ( ( j , h , k ) ) = 1 }
\end{array} \Longleftrightarrow \left\{\begin{array}{c}
\alpha((i, j, h))=1 \\
\text { and } \\
\alpha((i, h, k))=1
\end{array}\right.\right.
$$

\section{Proof:}

(1) is by convention.

(2), This application is similar to the proof of theorem (3.8).

(3)

$\Rightarrow)$ If we have $\alpha((i, j, k))=1$ and $\alpha((j, h, k))=1$.

We suppose that $\alpha((i, j, h))=0$, it means $A^{h j} \circ A^{j i}=B^{h i}$, so we have:

$A^{k h} \circ\left[A^{h j} \circ A^{j i}\right]=A^{k h} \circ B^{h i}=B^{k i}=\left[A^{k h} \circ A^{h j}\right] \circ A^{j i}=A^{k j} \circ A^{j i}=A^{k i}$.

Contradiction, thus $\alpha((i, j, h))=1$, the same ideas for $\alpha((i, h, k))=1$.

$\Leftarrow)$ If we have $\alpha((i, j, h))=1$ and $\alpha((i, h, k))=1$. 
We suppose that $\alpha((j, h, k))=0$ it means $A^{k h} \circ A^{h j}=B^{k j}$, so we have:

$A^{k h} \circ\left[A^{h j} \circ A^{j i}\right]=A^{k h} \circ A^{h i}=A^{k i}=\left[A^{k h} \circ A^{h j}\right] \circ A^{j i}=B^{k j} \circ A^{j i}=B^{k i}$.

Contradiction, thus $\alpha((j, h, k))=1$, use the same ideas to show $\alpha((i, j, k))=1$.

This completes the proof that if $\alpha$ corresponds to a category, it satisfies (1)-(3).

In the other direction, we will show that the associativity will be true under the assumption of these conditions. Any composition involving a morphism $B$ yields a morphism $B$, so we only need to check associativity for compositions of morphisms $A$. Furthermore, within a triple of objects $(i, j, k)$ condition (2) leads to one of the five isomorphism classes of categories in Theorem 4.2 and these are associative. Therefore it suffices to consider a distinct quadruple $(i, j, h, k)$.

1. If $\alpha((i, j, k))=0$ in this status we have four cases:

(a) Let $\alpha((i, j, h))=1$ and $\alpha((j, h, k))=1$, so $\alpha((i, h, k))=0$ because if not we get $\alpha((i, j, k))=1$ according to part (3) above.

Now

$A^{k h} \circ\left[A^{h j} \circ A^{j i}\right]=A^{k h} \circ A^{h i}=B^{k i},\left[A^{k h} \circ A^{h j}\right] \circ A^{j i}=A^{k j} \circ A^{j i}=B^{k i}$.

Thus, $A^{k h} \circ\left[A^{h j} \circ A^{j i}\right]=\left[A^{k h} \circ A^{h j}\right] \circ A^{j i}$.

(b) Let $\alpha((i, j, h))=1$ and $\alpha((j, h, k))=0$, then again $\alpha((i, h, k))=0$ and

$A^{k h} \circ\left[A^{h j} \circ A^{j i}\right]=A^{k h} \circ A^{h i}=B^{k i},\left[A^{k h} \circ A^{h j}\right] \circ A^{j i}=B^{k j} \circ A^{j i}=B^{k i}$. Thus, $A^{k h} \circ\left[A^{h j} \circ A^{j i}\right]=\left[A^{k h} \circ A^{h j}\right] \circ A^{j i}$.

(c) Let $\alpha((i, j, h))=0$ and $\alpha((j, h, k))=1$ then $A^{k h} \circ\left[A^{h j} \circ A^{j i}\right]=A^{k h} \circ B^{h i}=B^{k i},\left[A^{k h} \circ A^{h j}\right] \circ A^{j i}=A^{k j} \circ A^{j i}=B^{k i}$. Thus, $A^{k h} \circ\left[A^{h j} \circ A^{j i}\right]=\left[A^{k h} \circ A^{h j}\right] \circ A^{j i}$.

(d) Let $\alpha((i, j, h))=0$ and $\alpha((j, h, k))=0$ so;

$A^{k h} \circ\left[A^{h j} \circ A^{j i}\right]=A^{k h \circ B^{h i}}=B^{k i},\left[A^{k h} \circ A^{h j}\right] \circ A^{j i}=B^{k j} \circ A^{j i}=B^{k i}$. Thus, $A^{k h} \circ\left[A^{h j} \circ A^{j i}\right]=\left[A^{k h} \circ A^{h j}\right] \circ A^{j i}$. 
2. If $\alpha((i, j, k))=1$ so we have two cases:

(a) Let $\alpha((i, h, k))=0$, this case is similar to the case $(\alpha((i, j, k))=0)$.

(b) Let $\alpha((i, h, k))=1$ then by the equivalence (3) we are getting: either $\alpha((i, j, h))=0$ and $\alpha((j, h, k))=0$ in which case $A^{k h} \circ\left[A^{h j} \circ A^{j i}\right]=A^{k h} \circ B^{h i}=B^{k i},\left[A^{k h} \circ A^{h j}\right] \circ A^{j i}=B^{k j} \circ A^{j i}=B^{k i} ;$ or $\alpha((i, j, h))=1$ and $\alpha((j, h, k))=1$ in which case $A^{k h} \circ\left[A^{h j} \circ A^{j i}\right]=A^{k h} \circ A^{h i}=A^{k i},\left[A^{k h} \circ A^{h j}\right] \circ A^{j i}=A^{k j} \circ A^{j i}=A^{k i}$. Thus in both cases, $A^{k h} \circ\left[A^{h j} \circ A^{j i}\right]=\left[A^{k h} \circ A^{h j}\right] \circ A^{j i}$.

Finally, we will get to a reduced category.

\subsection{The upper and lower bounds of $\operatorname{Card}\left(M_{2}^{n}, r\right)$}

We refer to the definition of $\operatorname{Card}\left(M_{2}^{n}, r\right)$ and of $\operatorname{Card}\left(M_{2}^{n}, r, o\right)$, as in (2) we have the following equalities: $\operatorname{Card}\left(M_{2}^{n}, r, o\right) / n ! \leq \operatorname{Card}\left(M_{2}^{n}, r\right) \leq \operatorname{Card}\left(M_{2}^{n}, r, o\right)$. Consequently it suffices to obtain bounds for $\operatorname{Card}\left(M_{2}^{n}, r, o\right)$.

Theorem 5.2 The number of configurations of the function $\alpha$ on the triplet $[i, j, k]$ is 18 .

Proof: By example we work on the triplet $[1,2,3]$ in the case of the matrix $M_{2}^{3}$.

We have obtained 5 categories not isomorphic among themselves, that are associated to $M_{2}^{3}$, see the theorem (4.2).

Now we need to know how many there are up to ordered isomorphism, i.e. we don't confuse those that are similar. We can classify by their isomorphism classes denoted $\mathscr{A}_{1}, \mathscr{A}_{2}, \mathscr{A}_{3}, \mathscr{A}_{4}, \mathscr{A}_{5}$.

$\mathscr{A}_{1}$ : there is only one here.

$\mathscr{A}_{2}$ : For this to work we must choose a distinct ordered couple among 1,2 and 
3 so there are 6 choices.

$\mathscr{A}_{3}$ : For this to work we must choose a couple $(i j)$ but with the convention that $(i j)=(j i)$. We see then that the set of all responses is $\{(i j)$ and $(j i)\}$ so there are 3 choices.

$\mathscr{A}_{4}$ : For this to work we must choose a chain of 3 distinct elements, so there are $3 !=6$ choices.

$\mathscr{A}_{5}$ : This is a cycle that can go one way or the other then there are 2 choices:

$$
(12)+(23)+(31) \text { or }(13)+(32)+(21)
$$

Finally we are getting $1+6+3+6+2=18$ possibilities, so the number of ordered non-reduced categories is 18 , therefore $\operatorname{Card}\left(M_{2}^{3}, r, o\right)=18$.

Lemma 5.3 For $n \geq 3$ we have the following result:

$$
\operatorname{Card}\left(M_{2}^{n}, r\right) \leq \mathcal{C} \operatorname{ard}\left(M_{2}^{n}, r, o\right) \leq 18^{C_{n}^{3}}
$$

Indeed: On each distinct triplet $[i, j, k]$ we have shown that the number of ordered non-reduced categories is 18 see the theorem (5.2), so totally for these possibilities we get $18^{C_{n}^{3}}$. Not all of these choices satisfy the other axioms, but in any case this gives us $\mathcal{C}$ ard $\left(M_{2}^{n}, r, o\right) \leq 18^{C_{n}^{3}}$. As in $(2), \mathcal{C} \operatorname{Card}\left(M_{2}^{n}, r\right) \leq$ $\mathcal{C}$ ard $\left(M_{2}^{n}, r, o\right)$.

We now look for a lower bound.

Notation: We consider $X=\left\{x_{1}, \ldots, x_{n}\right\}$ the ordered set of $n$ objects. Let $P_{3}(X)$ be the set of parts having three elements in $X$, and $O_{3}(X)$ the set of distincts triplets (with an order that may be different from the order of $X$ ). We get $\operatorname{Card}\left(O_{3}(X)\right)=3$ ! $\operatorname{Card}\left(P_{3}(X)\right)$ and $\operatorname{Card}\left(P_{3}(X)\right)=C_{n}^{3}$. A triplet $\left(x_{i}, x_{j}, x_{k}\right) \in O_{3}(X)$ will be denoted still $(i, j, k)$, with $i \neq j, j \neq k$ and $i \neq k$.

Definition 5.4 Let $H \subset O_{3}(X)$ a subset. We say that $H$ is non-interfering if: 
for all $(i, j, k)$ in $H$, there isn't a triple of the form $(i, u, j)$ in $H$, nor a triple of the form $(j, v, k)$ in $H$.

Clearly:

Lemma 5.5 If $\mathrm{H} \subset \mathrm{O}_{3}(X)$ is a non-interfering subset, then for all $H^{\prime} \subset H$, we get that $H^{\prime}$ is also non-interfering.

Lemma 5.6 : If $H \subset O_{3}(X)$ is a non-interfering subset, there exists a unique category $\mathscr{A}_{H}$ in $M_{2}^{n}$ such that $\alpha(i, j, k)=1$ if $(i, j, k) \in H$ and $\alpha(i, j, k)=0$ if $(i, j, k) \notin H$.

Proof: we construct the category $\mathscr{A}_{H}$ corresponding to the function $\alpha$ given by the following conditions:

1. For a distinct triple, if $(i, j, k) \in H$ then $\alpha((i, j, k))=1$, and if $(i, j, k) \notin H$ so $\alpha((i, j, k))=0$.

2. The conditions of Theorem 5.1 are satisfied, notice that part (2) holds as well as part (3) because for a distinct quadruplet $i, j, h, k$ then: $\alpha((i, j, k))=1$ and $\alpha((j, h, k))=1 \Leftrightarrow \alpha((i, h, k))=1$ and $\alpha((i, j, h))=1$, indeed neither side can happen by the non-interfering condition.

Thus we get a category $\mathscr{A}_{H}$ by Theorem 5.1.

Corollary 5.7 If $\mathrm{H} \subset \mathrm{O}_{3}(X)$ is a non-interfering subset, then there is a different ordered reduced category $\mathscr{A}_{H^{\prime}} \in M_{2}^{n}$ for each different subset $H^{\prime} \subset H$. Accordingly, we get

$$
\mathcal{C a r d}\left(M_{2}^{n}, r, o\right) \geq 2^{\operatorname{Card}(H)}
$$

Theorem 5.8 By the lemma below we can determine the bounds for $\mathcal{C}$ ard $\left(M_{2}^{n}, r, o\right)$. We have the following bounds: 


$$
\frac{2^{[n / 3]^{3}}}{n !} \leq \operatorname{Card}\left(M_{2}^{n}, r\right) \leq 18^{C_{n}^{3}}
$$

where $n \geq 3$.

Proof: The upper bound is $18^{C_{n}^{3}}$ see the lemma (5.3). For the lower bound we will construct a non-interfering subset. Let $X=X_{1} \cup X_{2} \cup X_{3}$ be a disjoint union in other words with $X_{1} \cap X_{2}=\emptyset, X_{1} \cap X_{3}=\emptyset$, and $X_{2} \cap X_{3}=\emptyset$. So if we pose:

$$
H=\left\{(i, j, k) / x_{i} \in X_{1}, x_{j} \in X_{2} \text { and } x_{k} \in X_{3}\right\}
$$

the subset $H \subset O_{3}(X)$ becomes non-interfering.

Indeed, the conditions already give that $i \neq j, j \neq k$ and $i \neq k$ whenever $(i, j, k) \in H$, also we have no elements of the form $(i, u, j)$ or $(j, v, k)$ in $H$.

This set has $\operatorname{Card}(H)=\operatorname{Card}\left(X_{1}\right) \operatorname{Card}\left(X_{2}\right) \operatorname{Card}\left(X_{3}\right)$.

For example:

$\operatorname{Card}\left(X_{1}\right)=[n / 3], \operatorname{Card}\left(X_{2}\right)=[n / 3]$ and $\operatorname{Card}\left(X_{3}\right)=n-2[n / 3] \geq[n / 3]$.

Thus $\operatorname{Card}(H) \geq[n / 3]^{3}$ that is on the order of $n^{3} / 27$, and the lower bound is:

$$
\mathcal{C} \operatorname{ard}\left(M_{2}^{n}, r, o\right) \geq 2^{[n / 3]^{3}} .
$$

To remove the ordering, divide by $n$ ! as in (2).

Example 5.9 We showed earlier that $\operatorname{Card}\left(M_{2}^{3}, r\right)=5$, so for $n=3$ the above relation is realized:

$$
2 / 3=\frac{2^{[3 / 3]^{3}}}{3 !} \leq \operatorname{Card}\left(M_{2}^{3}, r\right)=5 \leq 18^{C_{3}^{3}}=18
$$

\section{Notation:}


Let $\sigma$ be given by the following notation:

$$
\sigma:=\lim _{n \rightarrow \infty} \sup \frac{\log \left(\operatorname{Card}\left(M_{2}^{n}, r\right)\right)}{n^{3}}
$$

Theorem 5.10 We can limit the constant $\sigma$ by:

$$
\frac{\log (2)}{27} \leq \sigma \leq \frac{\log (18)}{6}
$$

Proof: Apply Theorem (5.8).

\section{References}

[1] S. Allouch (2008). Sur l'existence d'une catégorie ayant une matrice strictement positive donnée. Preprint arXiv:0806.0060v1.

[2] S. Allouch (2010). On the existence of a category with a given matrix. Preprint arXiv:1007.2884.

[3] S. Allouch, C. Simpson (2014). Classification des matrices associées aux catǵories finies. Cah. Topol. Géom. Différ. Catég. 55:205-240.

[4] C. Berger, T. Leinster (2008). The Euler characteristic of a category as the sum of a divergent series. Homology, Homotopy Appl. 10:41-51.

[5] G. Brinkmann. B. McKay (2001). Posets on up to 16 Points. Order 19:147179.

[6] M. Cuntz. I. Heckenberger (2009). Weyl groupoids with at most three objects. J. Pure Appl. Alg. 213:1112-1128.

[7] T. Fiore, W. Lück. R. Sauer (2011). Finiteness obstructions and Euler characteristics of categories. Adv. in Math. 226:2371-2469. 
[8] T. Fiore, W. Lück. R. Sauer (2011). Euler characteristics of categories and homotopy colimits. Doc. Math. 16:301-354.

[9] M. Fleming. R. Gunther. R. Rosebrugh (2003). A Database of Categories. Journal of Symbolic Computation 35:127-135.

[10] M. Forrester-Barker (2002) Group objects and internal categories. Preprint arXiv:math/0212065v1.

[11] M. Jacobsen, J. Møller (2012). Euler characteristics of $p$-subgroup categories. J. Pure Appl. Alg. 216:2665-2696.

[12] M. Kapranov (1988). On the derived categories of coherent sheaves on some homogeneous spaces. Invent. Math. 92:479-508.

[13] T. Leinster (2008). The Euler characteristic of a category. Doc. Math. $13: 21-49$.

[14] K. Noguchi (2010). The Euler characteristic of infinite acyclic categories with filtrations. Preprint arXiv:1004.2547.

[15] A. Distler, T. Kelsey (2009). The monoids of orders eight, nine and ten. Annals of Mathematics and Artificial Intelligence 56:3-21. 